\title{
A WAY TO IMPROVE JET MODELING WITHIN RANS EQUATION SYSTEM
}

\author{
Alexey Troshin ${ }^{1,2}$ \\ ${ }^{1}$ Central Aerohydrodynamic Institute (TsAGI) \\ 1 Zhukovsky Street, Zhukovsky, Moscow Region, 140180, Russia \\ ${ }^{2}$ Moscow Institute of Physics and Technology (MIPT) \\ 9 Institutskiy per., Dolgoprudny, Moscow Region, 141700, Russia \\ e-mail: ai-troshin@yandex.ru
}

Keywords: Turbulence Model, Mixing Layer, Jet.

\begin{abstract}
Performance of several standard turbulence models in predicting the flow field of a plane jet is analyzed. Jet potential core length is shown to be overestimated by all the models considered. To solve the problem, an additional source term in the turbulence characteristic frequency $\omega$ equation is used. It accounts the longitudinal flow inhomogeneity and entrainment which influence turbulence in jet mixing layers. In comparison to the earlier publications on this source term, a coefficient in its formulation has been slightly altered to better predict round jets. The modified SSG/LRR- $\omega$ differential Reynolds stress model is used to compute several test cases including free subsonic plane and round jets, a supersonic underexpanded free round jet, and a coaxial jet. In all the cases, improvements over the standard SSG/LRR- $\omega$ and SST models are shown. Further steps involving RANS turbulence models verification with high order of accuracy LES computations are discussed.
\end{abstract}




\section{INTRODUCTION}

Today it is widely recognized that most turbulence models overestimate jet potential core

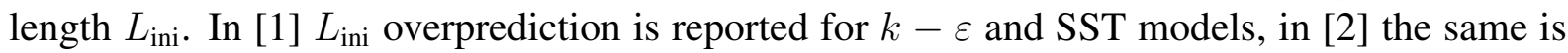
shown for an EARSM, and in [3] SSG/LRR- $\omega$ differential Reynolds stress model (DRSM) is found to give similar results. This issue can negatively influence the modeling of many problems involving civil aircraft propulsive jets, their interaction with nozzle walls and downstream structural elements of a plane. Several solutions of this problem have been proposed. In [1], $L_{\text {ini }}$ is reduced by taking into account the increase in turbulent diffusion intensity near the jet axis due to acoustic interaction between different parts of the mixing layer; in [4], another modification of turbulent diffusion is proposed compatible with the concept of mixing layer self-similarity. In [3], the problem is further investigated and another approach to improve the jet potential core modeling is developed which is not connected to the amplification of the modeled turbulence diffusion. Instead, the influence of longitudinal mean flow inghomogeneity on turbulence statistics is analyzed and taken into account. This approach is followed in the present paper. Firstly, more computational results confirming the inadequacies of standard models in predicting $L_{\text {ini }}$ are presented. After that, a minor update to one of the coefficients in the model proposed in [3] is made. Finally, new test cases computations using both modified and original models are reported.

The structure of the paper is as follows. In Section 2, the results of free plane jet computations using several standard turbulence models are compared, and common shortcomings of the solutions are discussed. In Section 3, a modification to the SSG/LRR- $\omega$ turbulence model is formulated and briefly commented. In Section 4, the computations of free subsonic plane and round jets, a supersonic underexpanded free round jet, and a coaxial jet are reported, and performance of the modified model is compared to the standard one and to the eddy viscosity SST model. Further steps in RANS turbulence models verification involving high order of accuracy LES computations are discussed in Section 5. The conclusions are made in Section 6.

\section{STANDARD TURBULENCE MODELS}

\subsection{Solver and turbulence models}

All the computations presented here are conducted using the EWT-TsAGI in-house code [5]. The solver uses structured multiblock hexahedral meshes. Hanging nodes at the block boundaries are allowed.

The following complete unsteady equation systems for the compressible air flow can be solved by the code:

- Euler equations;

- Navier-Stokes equations;

- Favre averaged Reynolds equations with one of the turbulence models listed below:

- Menter SST model [6];

— Coakley $q-\omega$ model [7];

- Spalart-Allmaras model [8];

— Wilcox Stress- $\omega$ DRSM [9];

— SSG/LRR- $\omega$ DRSM [10] and its modified version [3]. 
Second order of accuracy finite volume Godunov-Kolgan-Rodionov scheme is implemented in the solver. Explicit second order two step time marching (global, fractional, and local time steppings), first order backward Euler unconditionally stable implicit time marching (global and local time steppings), and second order dual time stepping are available.

The following notes concerning the numerical method need to be made. First, turbulence variables at a face of a cell are reconstructed with the same method (using van Leer limiter by default) as main variables thus maintaining the same accuracy order for all the equations. Second, exact iterative Godunov Riemann solver is used (turbulence variables are treated as passive scalars). To our experience, these features, aimed at increasing the accuracy of the method, generally do not degrade convergence.

Since the test cases reported here are steady, implicit scheme has been used in the computations.

\subsection{Test case specification}

To demonstrate the performance of the standard turbulence models, computations of a free subsonic plane jet of a cold air have been performed. Nozzle width $h$ based Reynolds number $\operatorname{Re}_{h}=u_{0} h / \nu$ is $6.7 \times 10^{6}$, Mach number is 0.30 . At the nozzle exit, approximately top hat velocity profile forms with thin turbulent boundary layers (each boundary layer has relative $0.99 u_{0}$-velocity width $\delta_{99} / h$ of 0.014 ). The primary role of these boundary layers is to supply the initial mixing layers with sufficient turbulence level for smooth self-similar development. On the other hand, both turbulence intensity and turbulent viscosity ratio in the potential core flow has been set negligibly small $\left(\left\langle u^{\prime}\right\rangle / u_{0} \sim \sqrt{k} / u_{0} \approx 10^{-5}\right.$, $\left.\omega h / u_{0} \sim 10^{-3}, \nu_{t} / \nu \sim 0.6\right)$.

Computational domain scheme and boundary conditions (BCs) are shown in Figure 1 (scaling is not proportional). Soft Riemann invariants based BCs are specified on the left boundary (above the jet flow region) and on the upper half of the right boundary. On the lower part of the right boundary, extrapolation BCs are used to better predict the outflow of the jet. Symmetry plane is set at the top (to avoid incorrect entrainment flow patterns) and the botoom (which is jet centerplane). External boundaries are placed $200 h$ away from the jet centerplane. No-slip $\mathrm{BCs}$ are set on the nozzle wall which length is equal to $h$. Ambient outer flow with relative velocity $u_{\infty} / u_{0}=0.01$ is specified in order to avoid instabilities due to interaction of soft BCs with stagnant air.

There are several published sets of experimental data on free subsonic plane jets. In this paper, data on velocity distributions along the centerline are taken from $[11,12,13,14]$. According to them, jet potential core length $L_{\text {ini }}$ defined as the distance from the nozzle exit where centerplane velocity is $0.99 u_{0}$, lies in the range $5 h \leq L_{\text {ini }} \leq 6 h$.

\subsection{Computational meshes and mesh convergence study}

A set of three nested meshes has been generated containing approximately 60000, 15000, and 4000 cells. An overview and nozzle region of the coarse mesh are presented in Figure 2. Hanging nodes outside the jet regions are clearly visible. They allow to significantly reduce the number of cells without affecting the accuracy of the results.

The fine mesh is designed to reproduce in details the mean flow in mixing layers (about 120 cells across the turbulent zone) and shear region downstream the initial region of the jet (about 140 cells per jet half-width). Nozzle boundary layer is resolved with 60 cells across it, the first cell height in wall units being of the order of 1 .

To study the mesh convergence, computations with SST turbulence model have been per- 


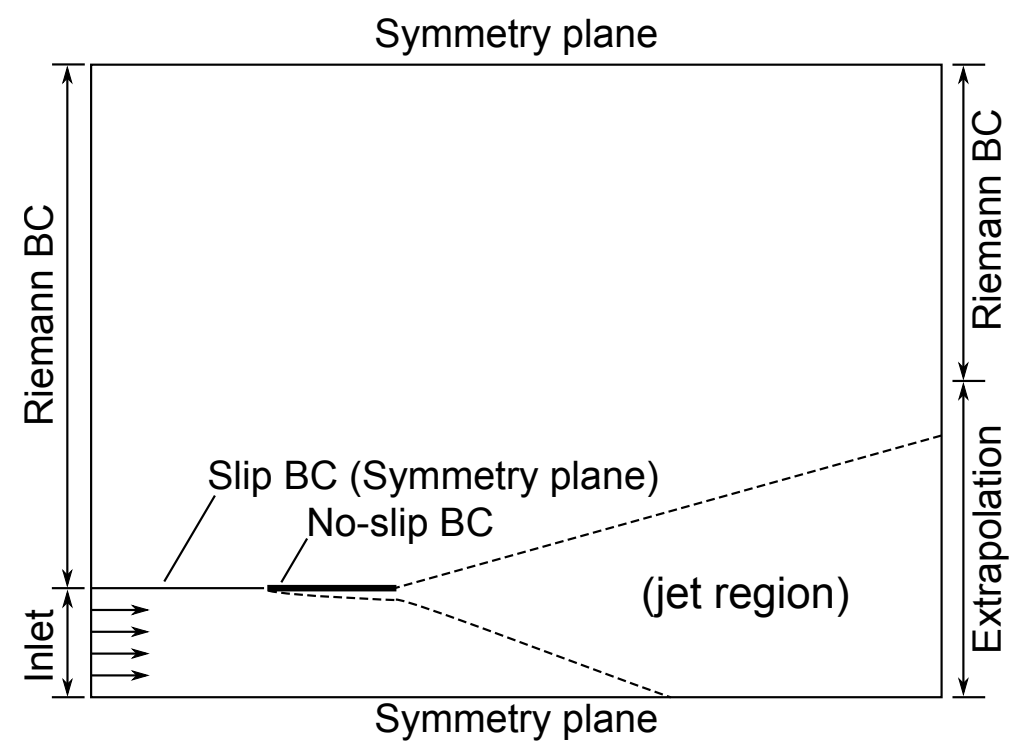

Figure 1: Computational domain scheme and boundary conditions of the plane jet test.
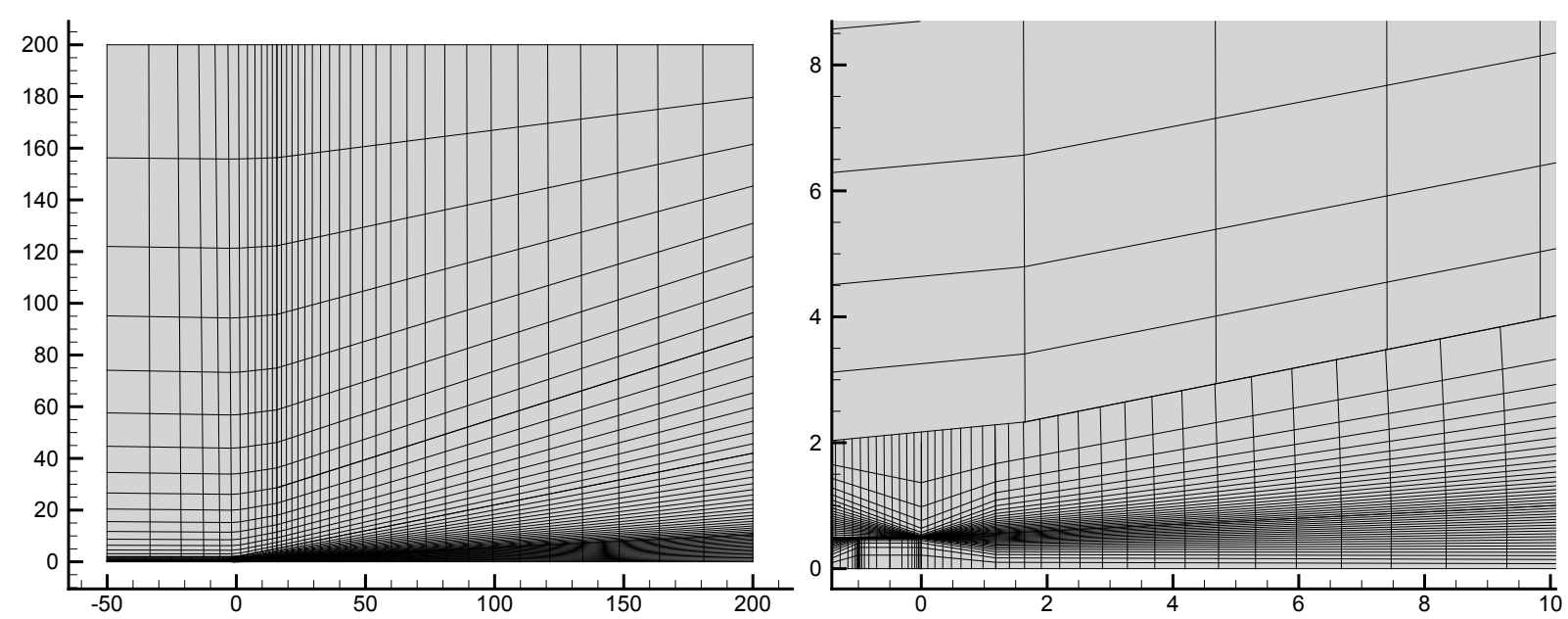

Figure 2: An overview (left) and nozzle region (right) of the coarse mesh for the plane jet test.

formed on each mesh. Resulting centerplane velocity and turbulent kinetic energy $k$ distributions in the region $0 \leq x / h \leq 30$ are shown in Figure 3 .

The difference in the velocity distributions is no more than $1.3 \%$ between the fine and medium meshes and no more than $2.6 \%$ between the medium and coarse meshes. Peak turbulent kinetic energies almost coincide, positions of $k$ maxima differing within $0.1 \%$ and $5 \%$, respectively. It is concluded that medium mesh is sufficient for further analysis in this paper. Another argument against the fine mesh is difficulties in obtaining the steady solution. KelvinHelmholtz type instability easily develops in the mixing layer, and to avoid it, it is necessary to start the computation with the first order numerical scheme, and after getting the steady field, proceed with second order scheme but with significantly limited time step. Even with these tricks, some travelling pressure waves persist is the mixing layers which slightly influence the centerplane velocity in the potential core. On the other hand, computations on the medium mesh converge to steady solutions smoothly. 

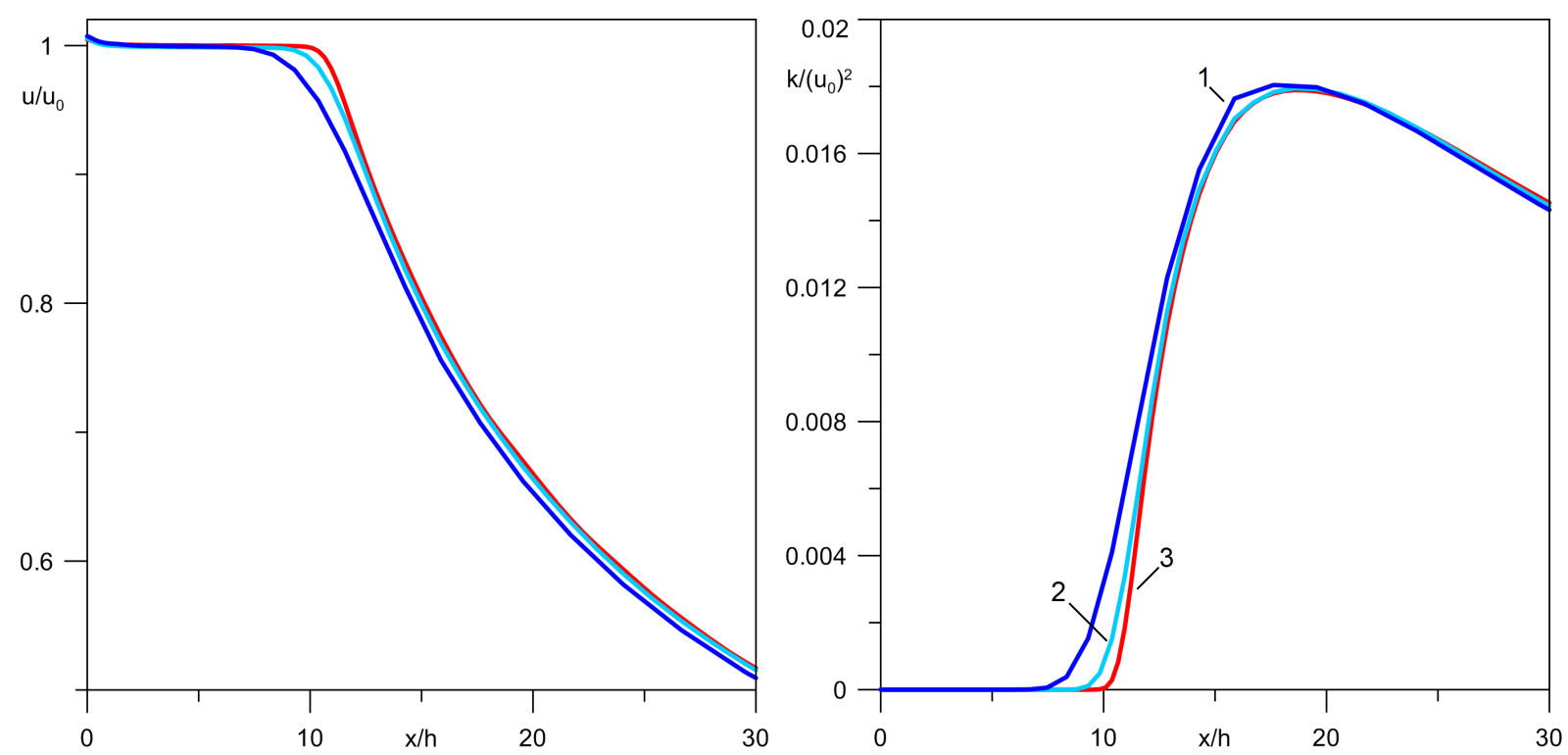

Figure 3: Centerplane velocity (left) and kinetic energy (right) distributions obtained on a set of nested meshes. 1 - coarse mesh, 2 - medium mesh, 3 - fine mesh.

\subsection{Results comparison}

After the mesh convergence study with SST model, computations with Spalart-Allmaras, Wilcox Stress- $\omega$, and SSG/LRR- $\omega$ models have been performed. In computations with DRSMs, isotropic turbulence has been specified at inflow boundaries. Centerplane velocity distributions obtained in these computations are compared with experimental data in Figure 4.

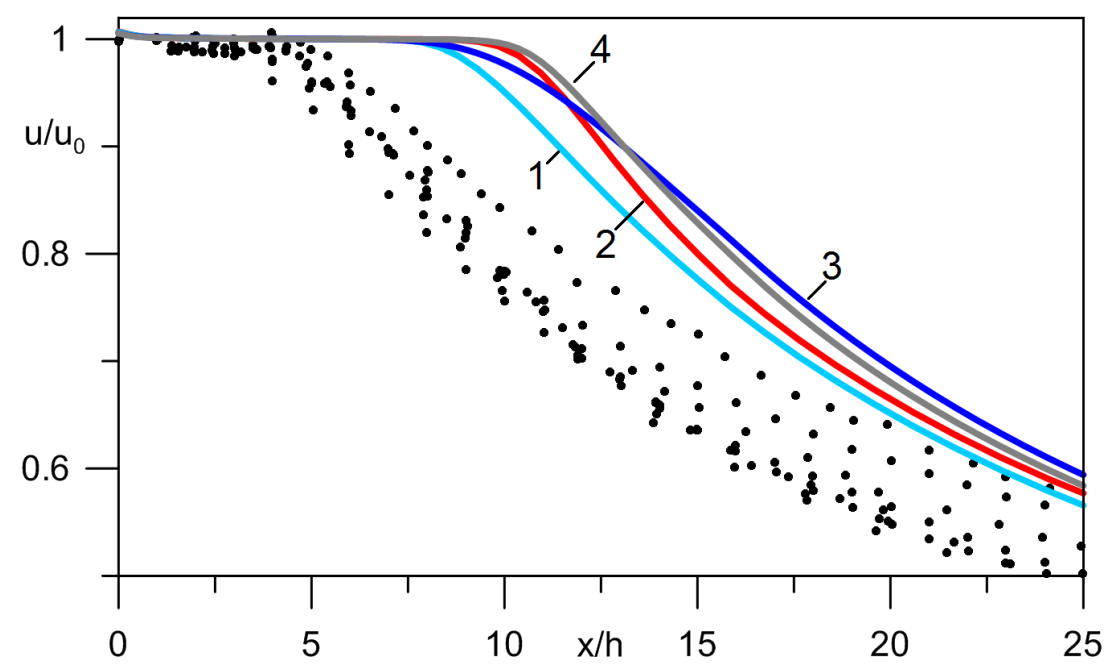

Figure 4: Centerplane velocity distributions obtained in plane jet test with different turbulence models. 1 Spalart-Allmaras model, 2 - SST, 3 - Stress- $\omega, 4$ - SSG/LRR- $\omega$.

It is clearly seen that all the models overestimate jet potential core length $L_{\text {ini }}$. The least error in $L_{\text {ini }}$ compared to the experimental data give Spalart-Allmaras model, the simplest among the models considered, and Stress- $\omega$ DRSM. They overpredict $L_{\text {ini }}$ by $60 \%$. The higher error is produced by SST and SSG/LRR- $\omega$ models (80\%). At the same time, mixing layer width growth rate $d D_{0.1} / d x\left(D_{0.1}\right.$ is the distance between the points where $u=0.1 u_{0}$ and $\left.u=0.9 u_{0}\right)$ is 
predicted much better and lies within $\pm 15 \%$ around experimentally observed value for all the turbulence models considered. Obtained values are collected in Table 1.

\begin{tabular}{ccc}
\hline Data & $L_{\text {ini }} / b$ & $d D_{0.1} / d x$ \\
\hline experiments & $5-6$ & $0.14-0.18$ \\
Spalart-Allmaras & 9 & 0.17 \\
SST & 10 & 0.15 \\
Stress- $\omega$ & 9 & 0.15 \\
SSG/LRR- $\omega$ & 10 & 0.14
\end{tabular}

Table 1: Plane jet potential core lengths and mixing layer width growth rates predicted by different turbulence models.

It turns out that different classes of turbulence models, from one equation eddy viscosity models to DRSMs, are affected by the same issue of overestimating $L_{\text {ini }}$ while correctly predicting $d D_{0.1} / d x$. This suggests an idea that mixing layer velocity profile is distorted such that the whole turbulent zone is "rotated" outwards the jet centerplane. In the following Section, a modification to the turbulence characteristic frequency $\omega$ equation is described which fixes the mixing layer velocity profile and recovers the correct $L_{\text {ini }}$ in computations.

\section{MODIFIED TURBULENCE MODEL}

The ideas of the modification are given here in brief and consecutive derivation of the formulas is omitted because these questions were the subjects of another paper [3] where they are described in details. The main goal of the current paper is to extend the number of test cases of the modified model and to more thoroughly assess its performance.

\subsection{The idea of the modification}

The first step of the SSG/LRR- $\omega$ model modification is recalibration of its coefficients in order to obtain as accurate description of mixing layers as possible. During the coefficients tuning it appears that temporal mixing layer velocity profile [15] is easy to reproduce, but single stream spatial mixing layer is a challenge. The process of tuning is described in [3]. The new "free stream" coefficient values using the designations of [10] are:

$$
\alpha_{\omega}=0.48, \quad \beta_{\omega}=0.0774, \quad D=0.20, \quad \sigma_{\omega}=0.659, \quad \sigma_{d}=0.373
$$

Velocity profiles in these two flows obtained after optimization of the coefficients are shown in Figure 5.

With the optimized coefficient set, temporal mixing layer fits the available data, but spatial mixing layer velocity profile is too sharp at the high velocity boundary of the mixing layer and too wide at the low velocity boundary. Indeed, this behavior is typical for most of the turbulence models. It leads to the idea that turbulence models do not take into account the influence of longitudinal flow inhomogeneity and entrainment effects in spatial mixing layer on turbulence. To describe these effects, a mechanism should be reproduced which increases the turbulent kinetic energy dissipation rate in the low velocity region of the spatial mixing layer and decreases it in the high velocity region by taking into account longitudinal gradients of different variables (velocity components $u_{i}$ and turbulence variables $\omega, \overline{u_{i}^{\prime} u_{j}^{\prime}}$, and its invariants). 

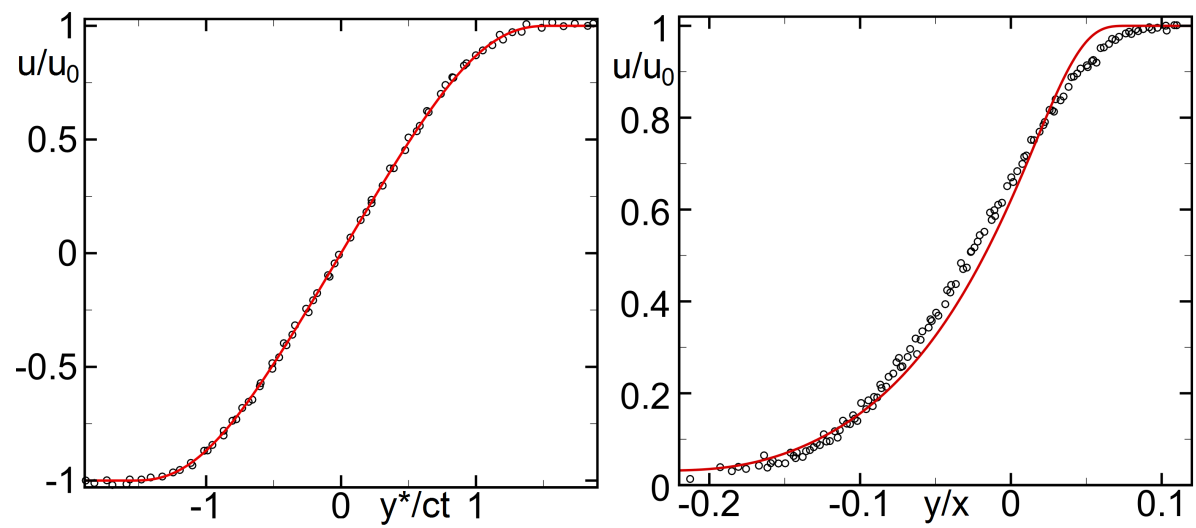

Figure 5: Velocity profiles in temporal (left) and single stream spatial (right) mixing layers after optimization of the SSG/LRR- $\omega$ model coefficients.

\subsection{Modified $\omega$ equation}

One possible form of taking into account the effects mentioned above is an additional source term $I_{\omega}$ in $\omega$ equation [3]. It can be written as

$$
\begin{array}{r}
\frac{\partial \bar{\rho} \omega}{\partial t}+\frac{\partial}{\partial x_{k}}\left(\bar{\rho} \omega \tilde{u}_{k}\right)=S_{\bar{\rho} \omega}+\bar{\rho} I_{\omega}, \\
I_{\omega}=-C_{\omega 3} 0.03 \operatorname{th}\left(\frac{2 \tilde{\Omega}_{i j}}{0.03 \omega^{4}} \frac{\partial \omega}{\partial x_{i}} \frac{\partial k}{\partial x_{j}}\right) \omega^{2}, \\
\tilde{\Omega}_{i j}=\frac{1}{2}\left(\frac{\partial \tilde{u}_{i}}{\partial x_{j}}-\frac{\partial \tilde{u}_{j}}{\partial x_{i}}\right), \quad C_{\omega 3}=20,
\end{array}
$$

where $S_{\bar{\rho} \omega}$ are the standard diffusion and source terms in $\omega$ equation.

$I_{\omega}$ is a Galilean invariant, local source term which takes different signs at the opposite edges of spatial mixing layers. In temporal mixing layer, it is zero. Recommended "free stream" $C_{\omega 3}$ value is 20 instead of previously reported value 22 to better capture the initial region of round supersonic jets. Near walls, $I_{\omega}$ is not used ("near-wall" value of $C_{\omega 3}$ is 0 ). The effect of $I_{\omega}$ inclusion in $\omega$ equation on single stream spatial mixing layer and on far field of an incompressible free plane jet is shown in Figure 6. Optimized coefficient set (1) was used in all these computations. It is seen that with $I_{\omega}$ term, the two flows considered fit the experimental data.

\section{TEST CASES}

The modified SSG/LRR- $\omega$ model as well as its original version and eddy viscosity SST model have been used to compute four jet test cases described below.

\subsection{Free subsonic plane jet}

The first test is a free subsonic plane jet described in Section 2. In addition to the results obtained above, modified SSG/LRR- $\omega$ model has been used. The same boundary conditions have been specified on the medium mesh. Resulting centerline velocity profiles are shown in Figure 7.

With the modified model, $L_{\text {ini }}$ is predicted to be $6 h$ which lies at the boundary of experimental range $5 h-6 h$ and is approximately two times lower than with two other models considered. Mixing layer width growth rate $d D_{0.1} / d x$ is 0.17 which is within experimental range. 

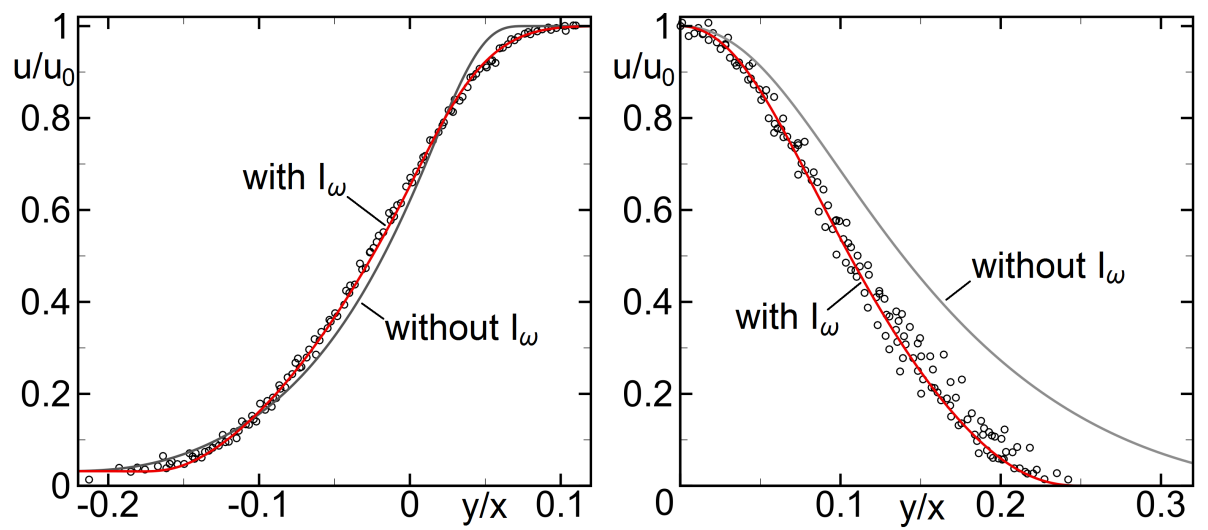

Figure 6: Velocity profiles in single stream spatial mixing layer (left) and plane jet (right) after optimization of the SSG/LRR- $\omega$ model coefficients with and without inclusion of $I_{\omega}$ term.

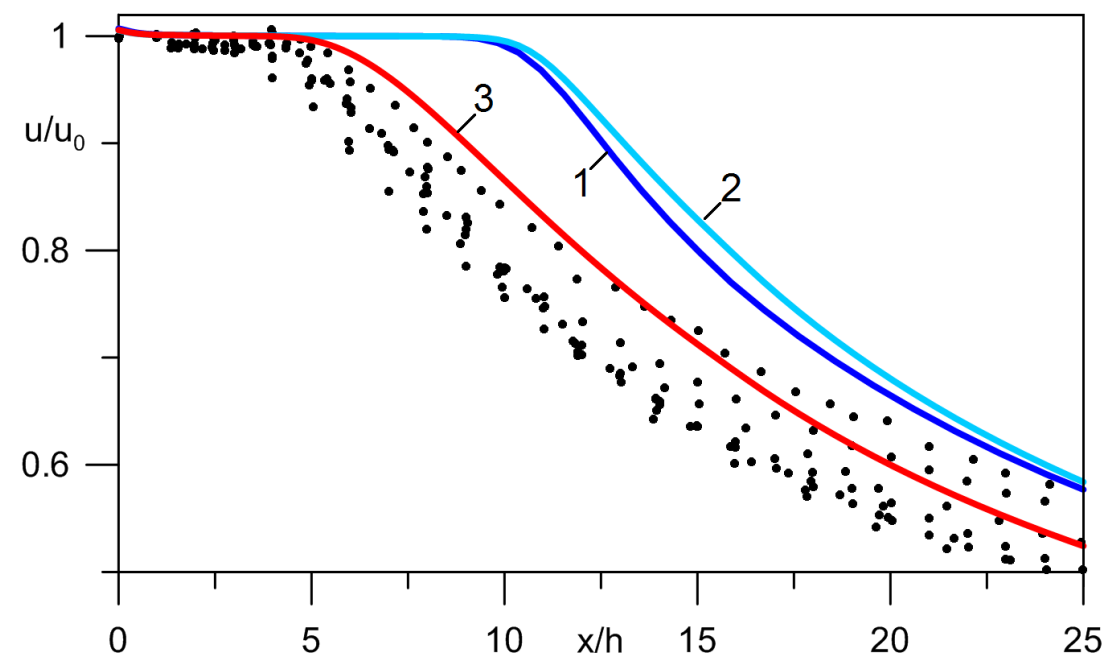

Figure 7: Centerplane velocity distributions obtained in plane jet test with: 1 - SST model, 2 - original SSG/LRR- $\omega$ model, 3 - its modified version.

\subsection{Free subsonic round jet}

This test case corresponds to the free subsonic plane jet described above except that computational mesh is a $5^{\circ}$ sector in azimuthal direction. In $(x, r)$ plane, the mesh coincides with the medium mesh used in the plane jet test. The resulting axial velocity distributions obtained with the three turbulence models are presented in Figure 8. Experimental values are taken from $[16,17,18]$. In round jets, experimental $L_{\text {ini }}$ values fall in the range $5 d-6 d$, where $d$ is nozzle diameter.

Again, the modified model gives $L_{\mathrm{ini}}=5 d$ which lies in the experimental range. The original SSG/LRR- $\omega$ model gives $L_{\text {ini }}=8.5 d$ and SST model gives $L_{\text {ini }}=8 d$. As in plane jet, mixing layer width growth rate fits the experimental data for all three turbulence models. Downstream the initial region, all three models predict too fast axial velocity decay. This is due to a well-known "round jet/plane jet anomaly" which can be eliminated by using either axial Pope correction or nonlinear pressure-strain models [19]. 


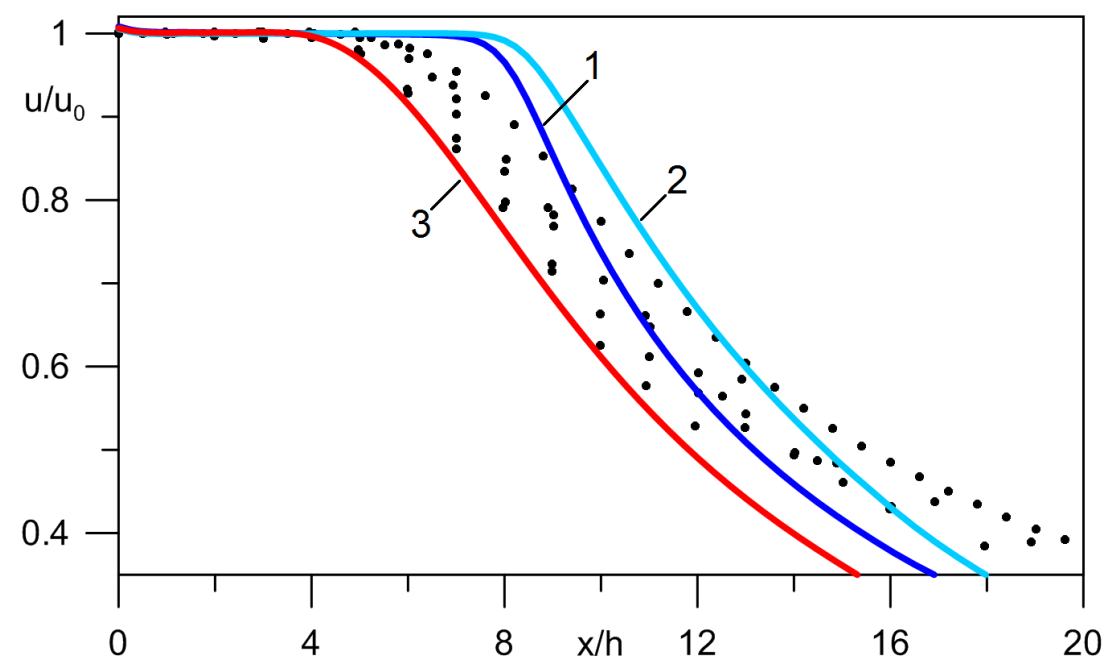

Figure 8: Axial velocity distributions obtained in round jet test with: 1 - SST model, 2 - original SSG/LRR- $\omega$ model, 3 - its modified version.

\subsection{Underexpanded free round jet}

In this test, a cold free supersonic round air jet has been modeled. It was studied experimentally at ITAM SB RAS [20]. The jet issues from a converging nozzle with Mach number at the exit equal to 1 . Nozzle pressure ratio is 2.8. Nozzle diameter $d$ based Reynolds number number $\operatorname{Re}_{d}=u_{e} d / \nu_{e}$ is $8.5 \times 10^{5}$, where parameters with subscript " $e$ " are taken at the nozzle exit. Turbulence level at the nozzle exit $\mathrm{Tu}_{e}$ is taken to be $0.9 \%$ to reproduce the experimental conditions.

An overview of the computational domain, mesh, and boundary conditions is shown in Figure 9. When generating the mesh, its density has been taken to be approximately the same as in medium mesh in free subsonic plane jet, which had been shown to produce mesh converged solutions.

In Figure 10, axial distributions of relative Pitot pressure $p_{t} / p_{0}$, where $p_{0}$ is the total pressure in the core of the nozzle, are shown. SST and original SSG/LRR- $\omega$ models capture the first 4 cells of the jet, after which they do not predict experimental $p_{t}$ fall. Moreover, SST model underpredicts the amplitude of $p_{t}$ oscillations at $x / d>5$. The modified SSG/LRR- $\omega$ model captures 5 jet cells and $p_{t}$ fall at $x / d>5$, which fits the experimental data better.

In Figure 11, three cross-sections of $p_{t} / p_{0}$ obtained in computations are compared with the experimental data. SST and original SSG/LRR- $\omega$ models give similar results predicting too sharp high velocity boundary of the mixing layer. The modified SSG/LRR- $\omega$ model make mixing layer smoother in this region and captures the experimental behavior of Pitot pressure.

\subsection{Coaxial jet}

This flow was also studied experimentally at ITAM SB RAS. Now it is one of the tests in First TILDA Workshop on Industrial LES \& DNS (2016). A cold free air jet issues from a dualstream round nozzle. The inner contour is subsonic with nozzle pressure ratio $\mathrm{NPR}_{1}=1.72$ and Mach number at the exit $M_{1}=0.8$. The outer contour is supersonic with $\mathrm{NPR}_{2}=2.25$ (underexpanded flow) and $M_{2}=1.0$. Outer contour diameter $D$ based Reynolds number $\operatorname{Re}_{D}=$ $u_{2} D / \nu_{2}$ is equal to $2.9 \times 10^{6}$.

Computational mesh near the nozzle is shown in Figure 12. As in previous test, mesh density 


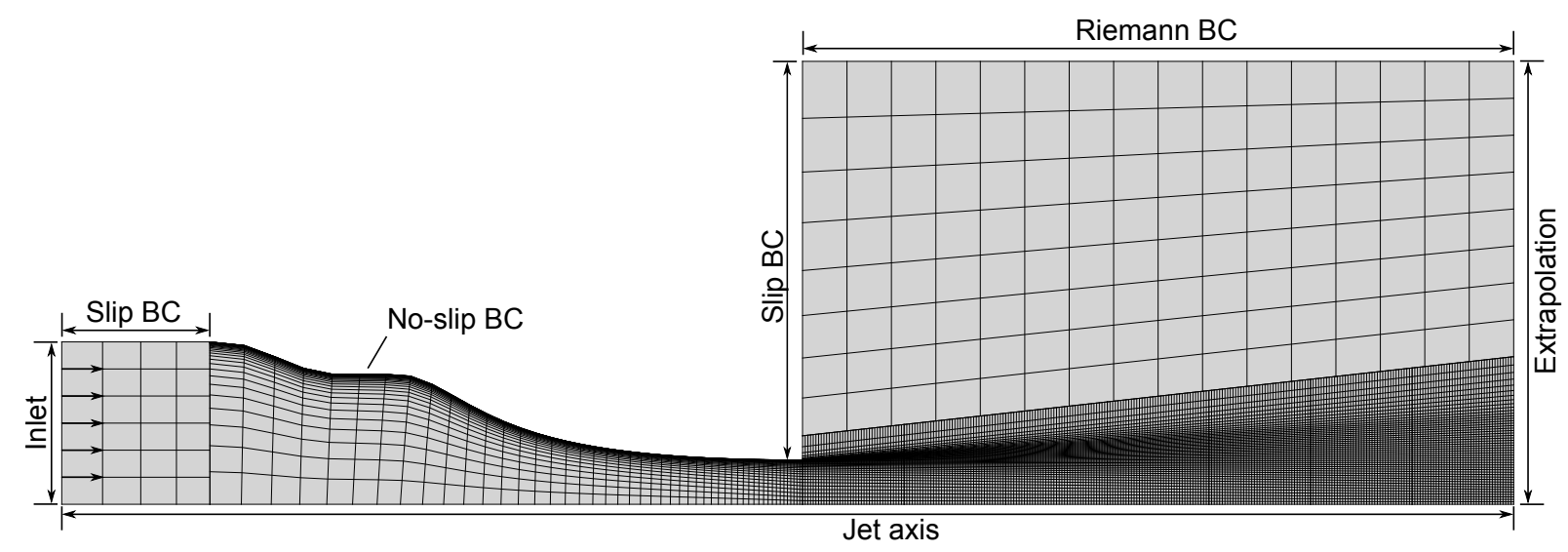

Figure 9: Computational domain, mesh, and boundary conditions in unedrexpanded free round jet test.

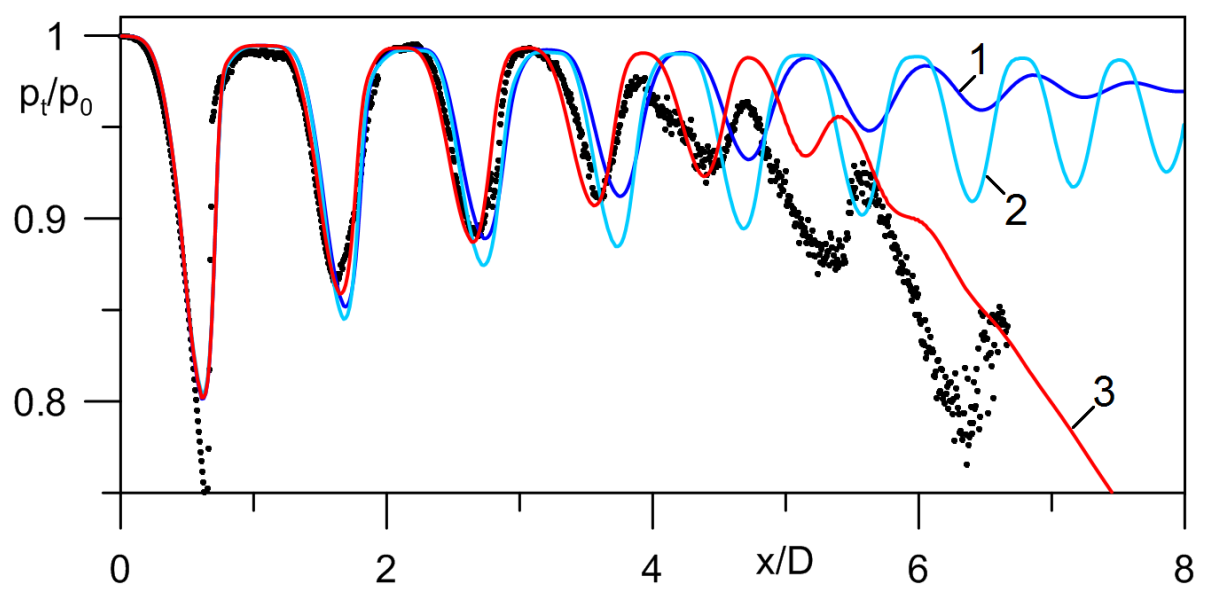

Figure 10: Axial distributions of $p_{t} / p_{0}$ in unedrexpanded free round jet test. $1-$ SST model, $2-$ original SSG/LRR- $\omega, 3$ - its modified version.
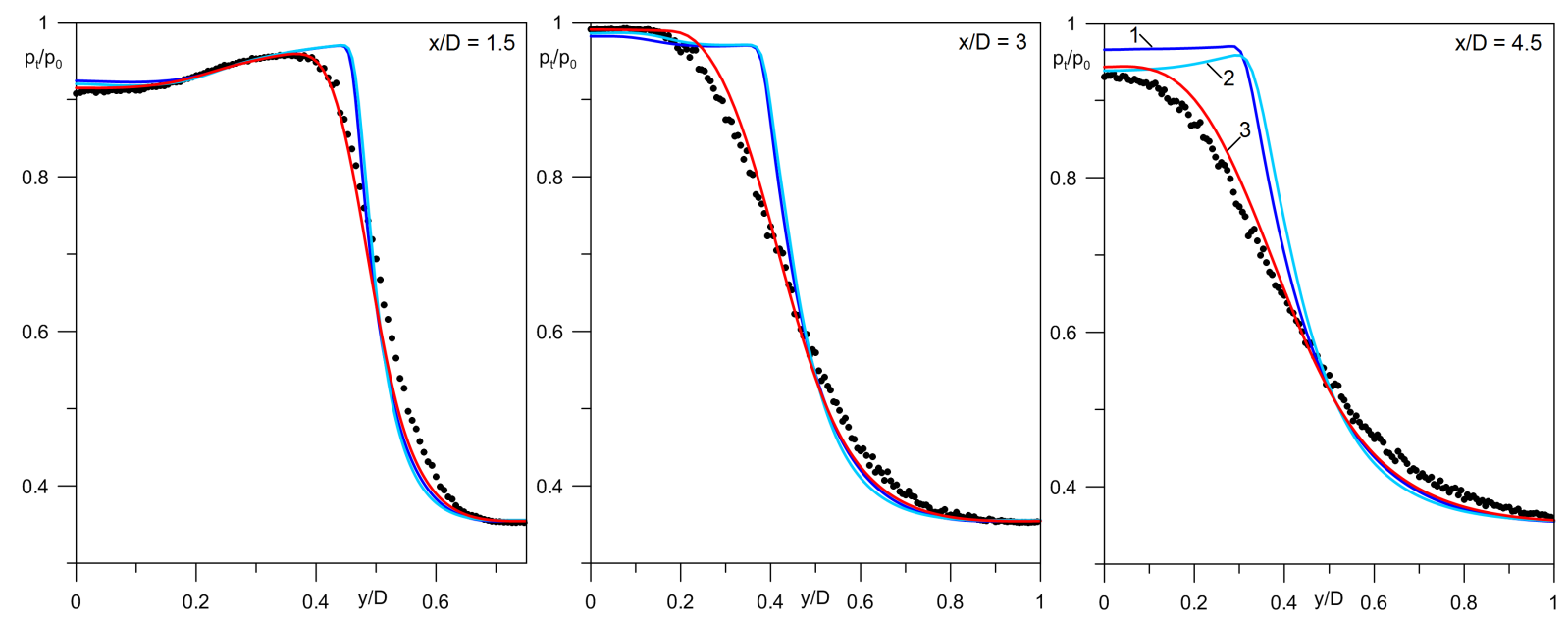

Figure 11: Cross-sections of $p_{t} / p_{0}$ in unedrexpanded free round jet test. 1 - SST model, 2 - original SSG/LRR$\omega, 3$ - its modified version. 
has been taken to be approximately the same as in medium mesh in free subsonic plane jet to get mesh converged solutions. In this test, it contains approximately 150000 cells.

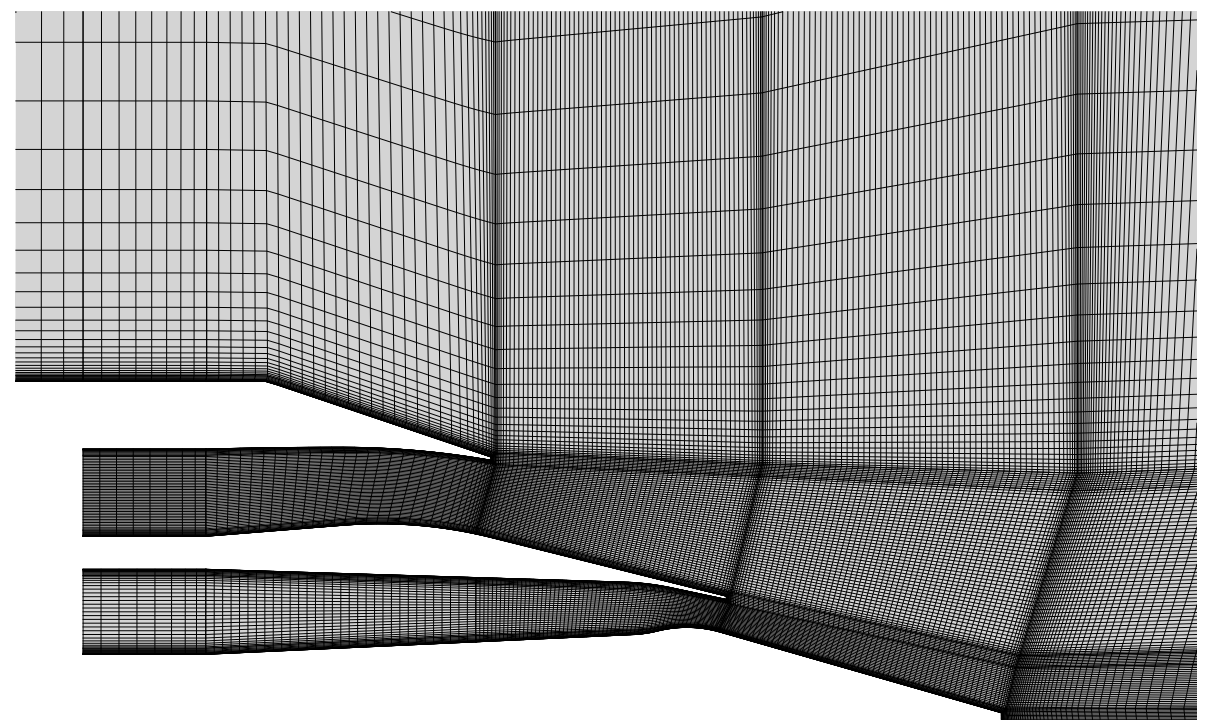

Figure 12: Mesh in coaxial jet test. Every second mesh line is shown.

In Figure 13, three computed cross-sections of $p_{t} / p_{0}$, where $p_{0}$ is the total pressure in the air supply of the outer contour, are compared with the experimental data. Longitudinal coordinate axis $x$ origin is at the tip of the central body. As clearly seen from the experimental data, boundary layers at the walls of the outer contour are much thicker than in computations. In fact, their traces collapse already at $x / D=0.08$. This leads to lower peak $p_{t}$ values at $y / D \approx$ 0.3 than in computations. SST model predicts a small region of $p_{t}$ decrease at $y / D \approx 0.2$ downstream the position $x / D=0.80$ which is absent both in experiments and in computations using DRSMs. On the other hand, wake intensity behind the central body is overpredicted by all the models considered. This is an interesting issue to investigate in the future. Apart from this discrepancies, the overall agreement between the computations and the experiment is satisfactory. The modified SSG/LRR- $\omega$ model predicts a different velocity profile in the outer mixing layer which seems to be realistic since similar behavior allowed to better fit the experimental data in the previous tests.

\section{FURTHER STEPS: LES BASED VALIDATION}

As has been shown in paper, the modified model improves modeling accuracy of the jet flows considered in the paper. However, several questions are still open, some of them are:

- Are there other, maybe simpler, forms of additional source term in $\omega$ equations which play the same role as $I_{\omega}$ used in the paper? If there are, which one to prefer?

- How to further modify turbulence model equations to better capture the decay of turbulent wakes, e.g. central body wake in the coaxial jet test?

- How do mixing layers develop when they originate from thick boundary layers? Are there any specific features? Does $I_{\omega}$ term needs a correction in this case? This can be studied in a series of coaxial jet test computations with correct boundary layers in the outer contour. 

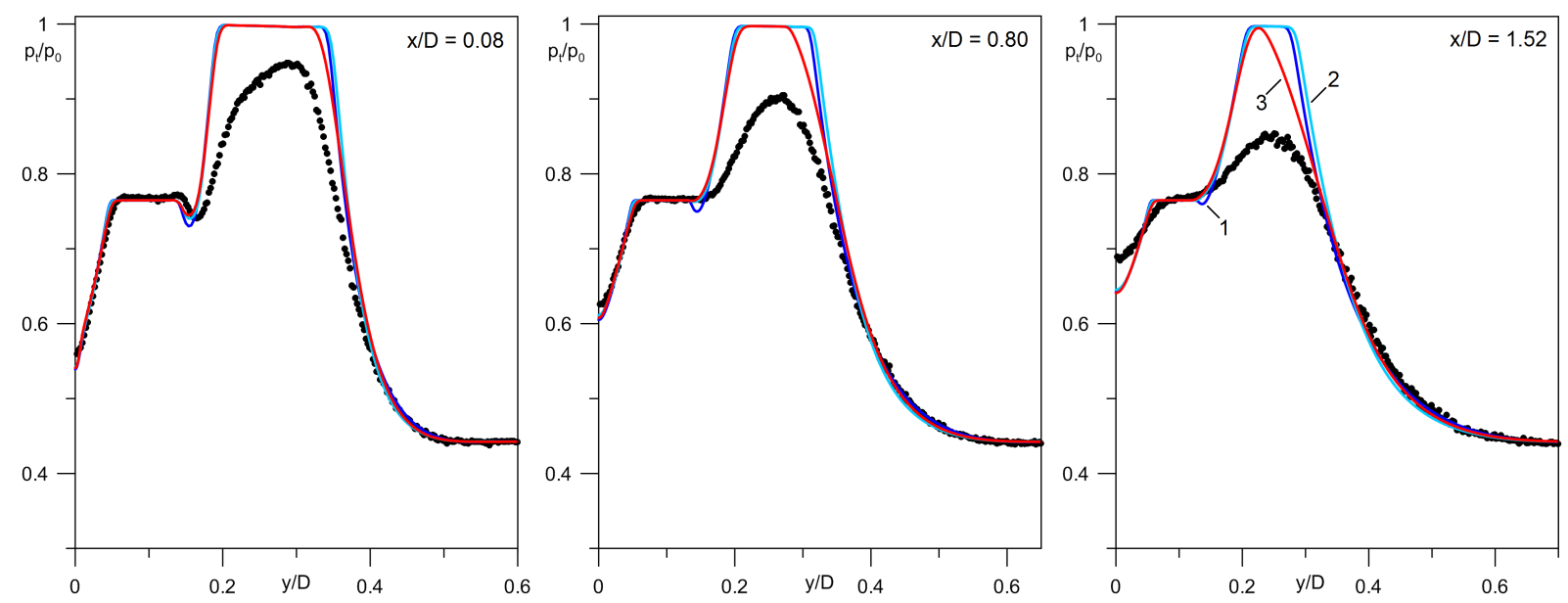

Figure 13: Cross-sections of $p_{t} / p_{0}$ in coaxial jet test. 1 - SST model, 2 - original SSG/LRR- $\omega, 3$ - its modified version.

Nowadays, there is strong progress in developing and validating the high order LES techniques, among which is Discontinuous Galerkin based LES [21]. These techniques are capable of accurately capturing turbulence phenomena in the range of scales, and it looks natural to use these capabilities for term-by-term validation and tuning of RANS turbulence models.

As the next step for the research presented in the paper, we plan to conduct LES based computations of the coaxial jet to get answers to at least some of the questions listed above. We have developed a new in-house code with the following features:

- High spatial order Discontinuous Galerkin method with up to piecewice cubic polynomials.

- Explicit time discretization (multistage Runge-Kutta schemes of up to $4^{\text {th }}$ order) with fractional time stepping technique.

- Complete compressible LES equation system with Smagorinsky subgrid scale model.

- MPI parallelism with efficient scalability on up to $\sim 10^{4}$ cores.

The data obtained in LES computations will allow to extract distributions of individual terms in Reynolds stress and $\omega$ equations. They will be compared to the data in RANS computations to improve the engineering models for different terms in RANS turbulence models.

\section{CONCLUSIONS}

It is shown that different standard turbulence models, from linear eddy viscosity models to DRSMs, incorrectly predict jet potential core length. SSG/LRR- $\omega$ DRSM is recalibrated and supplemented by an additional source term in the turbulence characteristic frequency $\omega$ equation which accounts the longitudinal flow inhomogeneity and entrainment. These effects influence turbulence in jet mixing layers and change its velocity profile. Four jet test cases are considered, in all of which the modified model improves the accuracy of flow field predicion over the standard models examined in the paper.

Theoretical part of the study was supported by RFBR, research project No. 16-38-00760.

Computations presented in the paper were supported by the Ministry of Education and Science, project No. 14.628.21.0005. 


\section{REFERENCES}

[1] W.A. Engblom, N.J. Georgiadis, A. Khavaran, Investigation of variable-diffusion turbulence model correction for round jets. 11th AIAA/CEAS Aeroacoustics Conference, Monterey, California, May 23-25, 2005.

[2] N.J. Georgiadis, D. Papamoschou, Computational investigations of high-speed dualstream jets. 9th AIAA/CEAS Aeroacoustics Conference and Exhibit, Hilton Head, South Carolina, May 12-14, 2003.

[3] A.I. Troshin, A turbulence model taking into account the longitudinal flow inhomogeneity in mixing layers and jets. 6th European Conference for Aerospace Sciences, Krakow, Poland, June 29 - July 3, 2015.

[4] A.I. Troshin, A turbulence model with variable coefficients for calculating mixing layers and jets. Fluid Dynamics, 47, No. 3, 320-328, 2012.

[5] V. Neyland, S. Bosniakov, S. Glazkov, A. Ivanov, S. Matyash, S. Mikhailov et al., Conception of electronic wind tunnel and first results of its implementation. Progress in Aerospace Sciences, 37, No. 2, 121-145, 2001.

[6] F.R. Menter, M. Kuntz, R. Langtry, Ten years of industrial experience with the SST turbulence model. Turbulence, Heat and Mass Transfer, 4, 625-632, 2003.

[7] T.J. Coakley, Turbulence modeling methods for the compressible Navier-Stokes equations. AlAA 16th Fluid and Plasma Dynamics Conference, Danvers, Massachusetts, July 12-14, 1983.

[8] P.R. Spalart, S.R. Allmaras, A one-equation turbulence model for aerodynamic flows. 30th Aerospace Sciences Meeting \& Exhibit, Reno, Nevada, January 6-9, 1992.

[9] D.C. Wilcox, Turbulence modeling for CFD. 3rd Edition. DCW Industries, 2006.

[10] R.-D. Cécora, R. Radespiel, B. Eisfeld, A. Probst, Differential Reynolds-stress modeling for aeronautics. AIAA Journal, 53, 739-755, 2015.

[11] E. Gutmark, I.J. Wygnanski, The planar turbulent jet. Journal of Fluid Mechanics, 73, 465-495, 1976.

[12] B.R. Ramaprian, M.S. Chandrasekhara, LDA measurements in plane turbulent jets. Journal of Fluids Engineering, 107, 264-271, 1985.

[13] M. Alnahhal, Th. Panidis, The effect of sidewalls on rectangular jets. Experimental Thermal and Fluid Science, 33, 838-851, 2009.

[14] R.C. Deo, J. Mi, G.J. Nathan, The influence of Reynolds number on a plane jet. Physics of Fluids, 20, 075108, 2008.

[15] J.H. Bell, R.D. Mehta, Development of a two-stream mixing layer from tripped and untripped boundary layers. AIAA Journal, 28, 2034-2042, 1990. 
[16] H.J. Hussein, S.P. Capp, W.K. George, Velocity measurements in a high-Reynoldsnumber, momentum-conserving, axisymmetric, turbulent jet. Journal of Fluid Mechanics, 258, 31-75, 1994.

[17] J.C. Lau, P.J. Morris, M.J. Fischer, Measurements in subsonic and supersonic free jets using a laser velocimeter. Journal of Fluid Mechanics, 93, 1-27, 1979.

[18] J. Bridges, M. Wernet, Establishing consensus turbulence statistics for hot subsonic jets. 16th AIAA/CEAS Aeroacoustics Conference, Stockholm, Sweden, June 7-9, 2010.

[19] K. Hanjalić, B. Launder, Modelling turbulence in engineering and the environment: second-moment routes to closure. Cambridge University Press, 2011.

[20] V.I. Zapryagaev, N.P. Kiselev, A.A. Pivovarov, Gasdynamic structure of an axisymmetric supersonic underexpanded jet. Fluid Dynamics, 50, No. 1, 87-97, 2015.

[21] F. Bassi, L. Botti, A. Colombo, A. Crivellini, A. Ghidoni, A. Nigro, S. Rebay, Time integration in the Discontinuous Galerkin code MIGALE - unsteady problems. Numerical Fluid Mechanics and Multidisciplinary Design, 128, 205-230, 2015. 